\title{
Dental Ethics - Ethik in der Zahnheilkunde
}

\author{
Dominik Groß • Ina Nitschke
}

Online publiziert: 2. Januar 2017

(C) Springer-Verlag Berlin Heidelberg 2017

Gibt es eine besondere Ethik für Zahnärzte? ${ }^{1}$ Nein, denn die grundlegenden, den Arzt verpflichtenden Prinzipen gelten auch für Zahnbehandler. Und doch sind wir der festen Überzeugung, mit dem vorliegenden Themenheft auf ein empfindliches (Forschungs-)Desiderat zu reagieren. Um diese Behauptung näher zu begründen, scheint es uns erforderlich, zwei konkrete Fragestellungen aufzugreifen:

- Was verstehen wir unter „Dental Ethics“ bzw. „Ethik in der Zahnheilkunde“ und

- gibt es Hinweise und Indikatoren für eine wachsende Relevanz dieses Themengebietes?

\section{Dental Ethics bzw. Ethik in der Zahnheilkunde - Versuche einer Begriffsbestimmung}

Mit dem noch wenig etablierten Syntagma „Ethik in der Zahnheilkunde“ wird diejenige Bereichsethik bezeichnet, die sich mit ethisch relevanten Fragen der Zahnheilkunde und ihrer Akteure (Zahnärzte mit ihren Teams, Patienten, berufsständische

\footnotetext{
1 In diesem Text wird das generische Maskulinum verwendet; des ungeachtet sind stets beide Geschlechter gemeint.
}

Prof. Dr. med. Dr. med. dent. Dr. phil. D. Groß $(\bowtie)$

Institut für Geschichte, Theorie und Ethik der Medizin, RWTH Aachen University,

Wendlingweg 2, 52074 Aachen, Deutschland

E-Mail: dgross@ukaachen.de

Prof. Dr. med. dent. I. Nitschke, MPH

Klinik für Alters- und Behindertenzahnmedizin, Zentrum für Zahnmedizin, Universität Zürich,

Plattenstrasse 15, 8032 Zürich, Schweiz 
Organisationen, Dentalindustrie etc.) beschäftigt. Im angloamerikanischen Sprachraum ist hierfür seit Jahrzehnten der Terminus „Dental Ethics“ etabliert. Demgegenüber hat der Arbeitskreis Ethik (AKE) der „Deutschen Gesellschaft für Zahn-, Mund- und Kieferheilkunde" (DGZMK) im Herbst 2010 mehrheitlich den Beschluss gefasst, für seinen Arbeitsbereich der Bezeichnung „Ethik in der Zahnheilkunde“ den Vorzug zu geben. Auf diese Weise sollen bereits auf terminologischer Ebene klinisch relevante Unterschiede zwischen dem deutschen Gesundheitssystem und den zahnärztlichen Versorgungssystemen der Länder des angloamerikanischen Sprachraums, aber auch augenfällige Differenzen in der beruflichen Selbstorganisation und im Selbstverständnis der jeweiligen zahnärztlichen Berufsgruppen deutlich gemacht werden (Groß 2012).

Wird die Zahnheilkunde als integraler Bestandteil der Heilkunde interpretiert, so dürfte die Ethik (in) der Zahnheilkunde mit einiger Berechtigung als Teilbereich innerhalb der Medizinethik gedeutet werden. Wer dagegen von den Professionen ausgeht, wird vermutlich eher zu der Erkenntnis gelangen, dass die Ethik in der Zahnheilkunde nicht (vollständig) in der Medizinethik aufgeht. Schließlich handelt es sich bei den Berufsgruppen - den Ärzten einerseits und den Zahnärzten andererseits - um zwei eigenständige, distinkte Professionen mit jeweils eigenen akademischen Studiengängen, berufsständischen Organisationen und (Muster-)Berufsordnungen. Für die letztgenannte Sichtweise findet sich auch das Argument, dass einige maßgebliche und prägende Themenbereiche der Medizinethik im zahnärztlichen Alltag ohne jede Relevanz sind (z. B. Stammzellforschung und IVF, Präimplantationsdiagnostik, Spätabbrüche, Organspende, Neuroenhancement, Sterbehilfe etc.) (Groß 2012).

Des ungeachtet spricht einiges gegen das vielzitierte pauschale Argument, dass die Zahnheilkunde kaum genuinen ethischen Klärungsbedarf biete, da der zahnärztliche Patient keinen vitalen Gefährdungen ausgesetzt sei. Klärungsbedürftige ethische Fragen und Dilemmata stellen sich nicht allein bei vitaler Bedrohung. Tatsächlich kommt einigen ethisch relevanten Themenbereichen in der Zahnheilkunde sogar eine besondere Bedeutung zu. Anders ausgedrückt: Die ethischen Herausforderungen, mit denen sich Zahnärzte in ihrer täglichen Berufspraxis konfrontiert sehen, sind in mancherlei Hinsicht sehr spezifisch. Sie offenbaren sich z. B. in einer erschwerten Kommunikation zwischen Zahnarzt und Patient: Der Oralbereich des Patienten ist sowohl der zentrale Bereich der Kommunikation als auch der Ort der Behandlung; er ist zudem beim Zahnarzt aus Gründen des Infektionsschutzes oft mit einem Mundschutz verdeckt. Ebenso stellt der Umgang - mit in der Zahnarztpraxis besonders zahlreichen - Angstpatienten („Dentophobien“) eine professionelle wie ethische Herausforderung dar.

Auch die Tatsache, dass einige schwerwiegende (systemische) Erkrankungen Frühmanifestationen in der Mundhöhle zeigen (z. B. Bulimie, Diabetes mellitus, bestimmte Formen der Leukämie) hat normative Implikationen, weist sie doch dem Zahnarzt eine besondere Verantwortung in der Frühdiagnostik zu, die weit über seine eigentliche fachliche Zuständigkeit hinausgeht. Ebenso diskussionswürdig sind gerechtigkeitsethische Fragen: So ist die Zahnheilkunde wie kaum ein anderes medizinisches Fach durch Zuzahlungsmodelle und Eigenanteile geprägt (,Zwei-KlassenMedizin“). Einige weitere Beispiele mit ethischen Implikationen werden im vor- 
liegenden Themenheft näher beleuchtet: etwa die boomenden Bereiche „Cosmetic Dentistry“ bzw. ,Wunscherfüllende Zahnheilkunde“, die höchst komplexe zahnärztliche Altersschätzung bei Flüchtlingen, die spezifischen normativen Implikationen der Alterszahnheilkunde und die besondere Relevanz zahnärztlicher Visualisierungsstrategien.

Auch ist die Freiberuflichkeit bei den Zahnärzten deutlich stärker ausgeprägt als bei der ärztlichen Berufsgruppe. Sie stellt, anders als bei den mehrheitlich angestellten bzw. beamteten Ärzten, die weit überwiegende Form der zahnärztlichen Berufsausübung dar, sodass beide Berufsstände z. T. sehr unterschiedliche Arbeitsbedingungen und -wirklichkeiten vorfinden, die wiederum spezifische ethische Implikationen in sich bergen.

Daneben existiert noch ein weiteres Argument für die These, dass die dentale Ethik nicht gänzlich in der ,klassischen“ Medizinethik aufgeht: So verlief die Aufwärtsentwicklung des Fachs Medizinethik an den deutschen medizinischen Fakultäten bisher weitgehend an der Zahnmedizin vorbei. Mit anderen Worten: Die Hochschulvertreter der Medizinethik wirkten bislang nur ausnahmsweise bzw. punktuell in die Zahnheilkunde hinein (Groß 2012). Dieser Aspekt führt unmittelbar zur zweiten hier adressierten Fragestellung:

\section{Gibt es Indikatoren für eine wachsende Relevanz dieses Themengebietes?}

Zunächst ist festzustellen, dass die Ethik in der Zahnheilkunde im deutschsprachigen Raum vor 2010 ein absolutes Schattendasein führte (Groß et al. 2009): So verzeichnete die medizinische Literaturdatenbank „Medline“ Mitte 2009 für den Zeitraum von Januar 2001 bis März 2009 bei der Eingabe des Schlagworts „Ethics, dental““ zwar 1009 Treffer; hierunter befanden sich jedoch lediglich vier deutschsprachige Publikationen. Eine ergänzende Recherche in der Bioethik-Datenbank „Belit“ erbrachte 2009 einen ähnlichen Befund: Sie wies für denselben Zeitraum gerade einmal zwei deutschsprachige Beiträge zum Themengebiet auf. In Deutschland gab es zu diesem Zeitpunkt weder ein Lehrbuch noch ein Themenheft zur Ethik in der Zahnheilkunde. Systematische Fortbildungskurse in diesem Fach fanden ebenso wenig statt wie gemeinsame Forschungsinitiativen von Zahnärzten und Bioethikern. Es existierte weder ein Lehrmodul zur Ethik in der Zahnheilkunde noch ein Lernzielkatalog, auf den man hätte zurückgreifen können.

Legt man den von dem US-amerikanischen Bioethiker David T. Ozar (2000) skizzierten dreistufigen Entwicklungsprozess des Fachs Dental Ethics zugrunde, so befand man sich im deutschsprachigen Raum um 2010 auf der niedrigsten Entwicklungsstufe: Ozar beschreibt diese Stufe als informelle Phase mit einer weitgehend ungeregelten fachbezogenen Ausbildung der Studierenden ohne definierte Lehrmaterialien; in den USA endete diese Stufe nach der Einschätzung Ozars bereits um 1980. Auch Wottrich (2010) diagnostizierte 2010 für die Ethik in der Zahnheilkunde in Deutschland im Vergleich mit der Ethik in der Medizin einen Entwicklungsrückstand von mehr als zwei Jahrzehnten. 
Tatsächlich gibt es viele Erklärungsversuche für diesen bis dahin höchst unbefriedigenden Befund:

Einen naheliegenden Erklärungsansatz bietet die Tatsache, dass die derzeit gültige zahnärztliche Approbationsordnung auf das Jahr 1955 (sic!) zurückgeht und insofern nach wie vor schlichtweg keine Ausbildung in ethischen Fragen vorsieht; es gibt dementsprechend (nahezu) keine Lehre(nde) in diesem Fach und damit fällt auch die wichtige bzw. prägende Funktion von Rollenmodellen weg.

Zudem werden die ethischen Implikationen der zahnärztlichen Tätigkeit traditionell ,kleingeredet“: So wird die Zahnheilkunde nicht selten als ,,reparative Disziplin mit sehr überschaubarem Anspruch“ (Weber 2002, S. 5) dargestellt, obwohl die Maßnahmen de facto weit mehr umfassen als die „Reparatur“ und „Wartung“ von zwei Zahnreihen.

Auch das im Zahnheilkundegesetz ${ }^{2}$ niedergelegte Krankheitsverständnis leistet einem derartigen Reduktionismus Vorschub: Es definiert Zahnkrankheit verkürzt als Normabweichung. Der Begriff „Patient“ tritt in jenem Gesetz lediglich an einer Stelle auf - und zwar in einem eher unbedeutenden Zusammenhang ( 1 Abs. 6: ,Anprobe von Bändern an Patienten“). Mit keinem Wort wird im Zahnheilkundegesetz das Leiden des Patienten angesprochen, mit keinem Begriff wird der Zahnarzt in seiner Eigenschaft als Heilperson adressiert.

Selbst in der zahnärztlichen Musterberufsordnung (MBO-Z) spielten ethische Belange bis 2010 keine (explizite) Rolle. So war und ist das Genfer Gelöbnis - die „moderne“ Version des Hippokratischen Eides - zwar traditionsgemäß der ärztlichen Musterberufsordnung (MBO-Ä) vorangestellt; dies galt aber nicht für die zahnärztliche MBO (MBO-Z). Auch fand der Begriff „Ethik“ in der MBO-Z bis dahin keinerlei Verwendung.

Seit 2010 ist jedoch in vielerlei Hinsicht ein Umdenken festzustellen, so dass es geboten erscheint, der ,Ethik in der Zahnheilkunde“ ein systematisches, disziplinäres Augenmerk zu schenken. Hierfür möchten wir sieben Trendindikatoren anführen:

1. Der im Mai 2010 verabschiedeten Fassung der Musterberufsordnung wurde erstmals das Genfer Gelöbnis beigestellt - im Sinne einer stillen Selbstverpflichtung des Berufsstandes und nach dem Vorbild der Ärzteschaft. Zudem tauchte in der MBO-Z nun erstmals der Begriff „Ethik“ auf, und zwar in $\S 2$ in der zentralen Aussage: „Insbesondere ist der Zahnarzt verpflichtet, a) seinen Beruf gewissenhaft und nach den Geboten der ärztlichen Ethik und der Menschlichkeit auszuüben [...]“" (Bundeszahnärztekammer 2010).

2. 2010 konnte zudem unter dem Dach der „Deutschen Gesellschaft für Zahn-, Mund- und Kieferheilkunde“ der nationale „Arbeitskreis Ethik“ gegründet werden, dem nach verhaltenem Start mittlerweile ca. 100 Mitglieder angehören darunter Hochschullehrer aus den Bereichen Medizinethik und Zahn-, Mundund Kieferheilkunde, aber auch an ethischen Fragen interessierte praktisch tätige

\footnotetext{
${ }^{2}$ Gesetz über die Ausübung der Zahnheilkunde in der Fassung der Bekanntmachung vom 16. April 1987 (BGB1. I S. 1225), das zuletzt durch Artikel 9 des Gesetzes vom 18. April 2016 (BGB1. I S. 886) geändert worden ist (2016). https://www.gesetze-im-internet.de/bundesrecht/zhg/gesamt.pdf. Zugegriffen: 6. Dez. 2016.
} 
Zahnärzte. Er bietet seitdem regelmäßige Fachtreffen und Fortbildungen an und entfaltet vielfältige wissenschaftliche bzw. publikatorische Aktivitäten.

3. Ebenfalls 2010 wurde an der ersten deutschen Universität - der RWTH Aachen im Rahmen der zahnärztlichen Ausbildung ein Pilot-Lehrprojekt „Dental Ethics“ initiiert. Es ist mittlerweile fester, obligater Bestandteil des ersten prothetischen Behandlungskurses. Zudem wurde 2011 im Rahmen der Jahrestagung des „Arbeitskreises für die Weiterentwicklung der Lehre in der Zahnmedizin“ (AKWLZ) der erste Fachvortrag zum Themenfeld „Dental Ethics“ registriert (AKWLZ 2011).

4. 2012 erschien sodann das erste deutschsprachige Lehrbuch „Ethik in der Zahnmedizin“, das u. a. 20 klinische Kasuistiken mit ethischem Klärungsbedarf enthält (Groß 2012) und zwischenzeitlich für Unterrichtszwecke, zum zahnärztlichen Selbststudium und bei zahnmedizinischen Teamfortbildungen eingesetzt wird.

5. Der seit 2012 erarbeitete und am 4. Juni 2015 verabschiedete „Nationale Kompetenzbasierte Lernzielkatalog Zahnmedizin“ (NKLZ) sieht erstmals in der Geschichte der zahnärztlichen Ausbildung einen Schwerpunkt in Ethik vor (Arbeitspaket 18). Insofern ist davon auszugehen, dass das betreffende Lehrfach mittelfristig flächendeckend unterrichtet werden wird.

6. Im November 2016 wurde erstmals - im Rahmen des „,Deutschen Zahnärztetages“ - ein „Dental Ethics Award“ für die beste Publikation im betreffenden Forschungsgebiet vergeben. Besagter Preis wurde vom AK Ethik der DGZMK gestiftet; er wird künftig jährlich vergeben und soll gezielte Anreize für Publikationen in diesem Bereich setzen.

7. Mit der vorliegenden Ausgabe von „Ethik in der Medizin“ erscheint das erste Themenheft zu „Dental Ethics - Ethik in der Zahnheilkunde“ in einer deutschsprachigen Fachzeitschrift. Zuvor war in dieser Zeitschrift in den Jahren 2015 und 2016 jeweils ein Fachbeitrag zu diesem Themengebiet erschienen; beide Beiträge zeigten bereits ein wachsendes Augenmerk für dentalethische Themen an; insofern erscheint das aktuelle Spezialheft gleichsam als logische Konsequenz dieser Entwicklung (Bettin et al. 2015; Groß et al. 2016).

Das vorliegende Themenheft unterstreicht somit den Trend sowie die Notwendigkeit einer tieferen Beschäftigung mit ethischen Fragestellungen in der Zahnheilkunde. Ihm soll daher unser abschließendes Augenmerk gelten:

„Dental Ethics - Ethik in der Zahnheilkunde“ versammelt neben einem Tagungsbericht insgesamt fünf Originalarbeiten zu höchst verschiedenen normativ relevanten Aspekten der zahnärztlichen Tätigkeit. Den Anfang macht ein Aufsatz von Mathias Wirth, Christoph L. Menzel, Daniela Chan Mi Lee und Heinz-Peter Schmiedebach. Die Autoren widmen sich einer gesellschaftspolitisch höchst aktuellen außertherapeutischen Aufgabe der Zahnärzteschaft: Der zahnärztlichen Altersschätzung bei jungen Flüchtlingen mittels Röntgendiagnostik und den damit verbundenen ethischen Fragen. Sie arbeiten heraus, dass die Altersbestimmung von minderjährigen, unbegleiteten Flüchtlingen durch Zahnärzte ,zum Mechanismus des othering des ohnehin Fremden“ führt und dem zahnärztlichen Heilauftrag unter Umständen diametral entgegensteht. 
Anschließend richten Dirk Leisenberg und Dominik Groß in ihrem Beitrag über neue Visualisierungsstrategien in der Zahnheilkunde den Fokus auf zahnärztliche Aufklärungsgespräche und den potentiell direktiven bzw. manipulativen Einsatz von Visualisierungstechniken. Sie gelangen zu dem Schluss, dass Visualisierungen nur bei vorsichtigem, überlegtem und selbstkritischem Einsatz und einer flankierenden spezifischen Aufklärung das Potential bieten, die Selbstbestimmung des Patienten zu stärken und den Rahmenbedingungen eines „Informed consent“ zu entsprechen.

Es folgt ein Beitrag der Medizinethiker Gerald Neitzke und Bernd Oppermann. Sie nehmen das Themenfeld „Wunscherfüllende“ bzw. „Kosmetische Zahnheilkunde" in den Blick. Dabei arbeiten sie heraus, dass die Indikation das zentrale zahnärztliche Kriterium für bzw. gegen ästhetisch motivierte Behandlungswünsche darstellt.

Die Medizinsoziologen Mike Jacob und Michael Dick widmen sich sodann dem Themenfeld zahnärztliche Misserfolge. Sie stellen die Methode des Triadengesprächs vor und erörtern, inwieweit und auf welche Weise mit dieser Methode ein verändertes Verständnis des zahnärztlichen Misserfolgs sowie eine positive Fehlerkultur - namentlich eine Offenheit für das Lernen aus Misserfolgen - möglich wird.

Am Ende steht ein stark praxisorientierter Beitrag von Ina Nitschke, Dominik Groß und Julia Kunze. Sie diskutieren sowohl auf der theoretischen Ebene als auch anhand konkreter Patientenfälle die spezifischen normativen Bedarfe (hoch)betagter bzw. dementer zahnärztlicher Patienten. Dabei arbeiten sie heraus, dass in der Zahnmedizin für geriatrische Patienten in vielen Fällen eine „Kompromissbehandlung“ erfolgen muss, die abweichenden Regeln folgt und insofern besondere kommunikative wie auch normative Anforderungen stellt.

\section{Literatur}

AKWLZ (2011) 3. Jahrestagung. https://www.akwlz.de/3.-jahrestagung-2011. Zugegriffen: 5. Dez. 2016 Bettin H, Spassov A, Werner MH (2015) Asymmetrien bei der Einschätzung des kieferorthopädischen Behandlungsbedarfs. Ethik Med 27:183-196

Bundeszahnärztekammer (2010) Musterberufsordnung der Bundeszahnärztekammer. http://213.196.235. 25/rechtsgrund/MBO_20100519.pdf. Zugegriffen: 6. Dez. 2016

Groß D (2012) Ethik in der Zahnmedizin. Ein praxisorientiertes Lehrbuch mit 20 kommentierten klinischen Fällen. Quintessenz, Berlin

Groß D, Wolfart S, Schäfer G (2009) „Ethik in der Zahnheilkunde“ im internationalen Vergleich. Analysen, Konzepte, Initiativen. Dtsch Zahnärztl Z 64(7):410-418

Groß D, Lenk C, Utzig B (2016) Normative Rahmenbedingungen der Rekrutierung und Nutzung extrahierter Zähne in Forschung und Lehre. Ethik Med 28:21-31

Ozar DT (2000) Dental ethics as an intellectual discipline - taking the next step. J Am Coll Dent 67(1):30-34

Weber H (2002) Geleitwort. In: Groß D (Hrsg) Zwischen Theorie und Praxis 3: Ethik in der Zahnheilkunde. Königshausen \& Neumann, Würzburg, S 5-6

Wottrich S (2010) Ethik und Recht im Studium der Zahnmedizin und im beruflichen Alltag. Dissertation. Medizinische Hochschule, Hannover 